\title{
Existence and Uniqueness Theorems for Generalized Set Differential Equations
}

\author{
Andrej Plotnikov, ${ }^{1,2}$, Natalia Skripnik ${ }^{1}$ \\ ${ }^{1}$ Department of Optimal Control \& Economic Cybernetics, Odessa National University named after I.I. Mechnikov, Odessa, 65026, \\ Ukraine \\ ${ }^{2}$ Department of Applied Mathematics, Odessa State Academy of Civil Engineering and Architecture, Odessa, 65029, Ukraine
}

\begin{abstract}
In this paper the concept of generalized differentiability for set-valued mappings proposed by A.V. Plotnikov, N.V. Skripnik is used. The generalized set-valued differential equations with generalized derivative are considered and the existence and uniqueness theorems are proved.
\end{abstract}

Keywords set-valued mapping, generalized derivative, existence and uniqueness theorems

\section{Introduction}

The concept of derivative for set-valued mapping was first entered by M. Hukuhara[1]. Then the problems of differentiability of fuzzy mappings were considered by T. F. Bridgland[2], J.N. Tyurin[3], H.T. Banks and M.Q. Jacobs[4], A.V. Plotnikov[5, 6], A.N. Vityuk[7], B. Bede and S.G. Gal[8], A.V. Plotnikov and N.V. Skripnik[9]. The properties of the these derivatives were considered in[10-18].

F.S. de Blasi and F. Iervolino begun studying of set-valued differential equations (SDEs) in semilinear metric spaces[12,19-21]. Now it developed in the theory of SDEs as an independent discipline. The properties of solutions, the impulsive SDEs, control systems and asymptotic methods for SDEs were considered[5,6,9-11,16-24]. On the other hand, SDEs are useful in other areas of mathematics. For example, SDEs are used as an auxiliary tool to prove the existence results for differential inclusions. Also, one can employ SDEs in the investigation of fuzzy differential equations. Moreover, SDEs are a natural generalization of usual ordinary differential equations in finite (or infinite) dimensional Banach spaces[19].

In[9] a new concept of a derivative of a set-valued mapping that generalizes the concept of Hukuhara derivative was entered and a new type of a set-valued differential equation such that the diameter of its solution can whether increase or decrease (for example, to be periodic) was considered. In the ideological sense this definition of the derivative is close to the definitions proposed in $[5,6,8]$.

* Corresponding author:

a-plotnikov@ukr.net (Andrej Plotnikov)

Published online at http://journal.sapub.org/control

Copyright (C) 2012 Scientific \& Academic Publishing. All Rights Reserved
In this paper the generalized set-valued differential equations with generalized derivative are considered and the existence and uniqueness theorems are proved.

\section{The Generalized Derivative}

Let $\operatorname{conv}\left(R^{n}\right)$ be a space of all nonempty convex closed sets of $R^{n}$ with Hausdorff metric

$$
h(A, B)=\min \left\{r \geq 0: A \subset B+S_{r}(0), B \subset A+S_{r}(0)\right\},
$$

where $A, B \in \operatorname{conv}\left(R^{n}\right), \quad S_{r}(0)=\left\{s \in R^{n}:\|s\| \leq r\right\}$.

Definition 1[1]. Let $X, Y \in \operatorname{conv}\left(R^{n}\right)$. A set $Z \in \operatorname{conv}\left(R^{n}\right)$ such that $X=Y+Z$ is called a Hukuhara difference of the sets $X$ and $Y$ and is denoted by $X-{ }_{-}^{h} Y$.

From Rådström's Embedding Lemma[25] it follows that if this difference exists, then it is unique.

Let $I=\left[t^{\prime}, t^{\prime \prime}\right] \subset R ; X: I \rightarrow \operatorname{conv}\left(R^{n}\right)$ be a set-valued mapping; $\left(t_{0}-\Delta, t_{0}+\Delta\right) \subset I$ be a $\Delta$-neighbourhood of a point $t_{0} \in I ; \Delta>0$.

For any $t \in\left(t_{0}-\Delta, t_{0}+\Delta\right)$ consider the following Hukuhara differences if these differences exist.

$$
\begin{aligned}
& X(t) \frac{h}{-} X\left(t_{0}\right), t \geq t_{0} \\
& X\left(t_{0}\right) \stackrel{h}{-} X(t), t \geq t_{0} \\
& X\left(t_{0}\right) \stackrel{h}{-} X(t), t \leq t_{0} \\
& X(t) \stackrel{h}{-} X\left(t_{0}\right), t \leq t_{0}
\end{aligned}
$$

The differences (1) and (2)[(3) and (4)] are called the right[left] differences. From the definition of the Hukuhara difference it follows that both one-sided differences exist only in the case when $X(t) \equiv F+\{f(t)\}$ for $t \in\left[t_{0}, t_{0}+\Delta\right)$ or $t \in\left(t_{0}-\Delta, t_{0}\right]$. If all differences (1)-(4) exist then 
$X(t) \equiv F+\{f(t)\} \quad$ in $\Delta$-neighbourhood of the point $t_{0}$.

If for all $t \in\left(t_{0}-\Delta, t_{0}+\Delta\right)$ there exists only one of the one-sided differences, then using the properties of the $\mathrm{Hu}$ kuhara difference, we get that the mapping $\operatorname{diam} X: I \rightarrow R_{+}$in the $\Delta$-neighbourhood of the point $t_{0}$ can be:

a) non-decreasing on $\left(t_{0}-\Delta, t_{0}+\Delta\right)$;

b) non-increasing on $\left(t_{0}-\Delta, t_{0}+\Delta\right)$;

c) non-decreasing on $\left(t_{0}-\Delta, t_{0}\right)$ and non-increasing on $\left(t_{0}, t_{0}+\Delta\right)$

d) non-increasing on $\left(t_{0}-\Delta, t_{0}\right)$ and non-decreasing on $\left(t_{0}, t_{0}+\Delta\right)$.

Hence, for each of the above mentioned cases only one of combinations of differences is possible:

a) (1) and (3); b) (2) and (4); c) (2) and (3); d) (1) and (4).

Consider four types of limits corresponding to one of the difference types:

$$
\begin{aligned}
& \lim _{t \rightarrow t_{0}^{+}} \frac{1}{t-t_{0}}\left(X(t) \frac{h}{X}\left(t_{0}\right)\right) ; \\
& \lim _{t \rightarrow t_{0}^{+}} \frac{1}{t-t_{0}}\left(X\left(t_{0}\right) \frac{h}{-} X(t)\right) ; \\
& \lim _{t \rightarrow t_{0}^{-}} \frac{1}{t_{0}-t}\left(X\left(t_{0}\right) \frac{h}{-} X(t)\right) ; \\
& \lim _{t \rightarrow t_{0}^{-}} \frac{1}{t_{0}-t}\left(X(t) \frac{h}{-} X\left(t_{0}\right)\right) .
\end{aligned}
$$

So it is possible to say that in the point $t_{0}$ not more than two limits can exist (as we assumed that there exist only two of four Hukuhara differences).

Considering all above we have that there can exist only the following combinations of limits:

$$
\begin{aligned}
& \text { a) (5) and (7); b) (6) and (8); } \\
& \text { c) (6) and (7); d) (5) and (8). }
\end{aligned}
$$

Definition 2[9]. If the corresponding two limits exist and are equal we will say that the mapping $X(\cdot)$ is differentiable in the generalized sense in the point $t_{0}$ and denote the generalized derivative by $D X\left(t_{0}\right)$.

Let us say that the set-valued mapping $X: I \rightarrow \operatorname{conv}\left(R^{n}\right)$ is differentiable in the generalized sense on the interval $I$ if it is differentiable in the generalized sense at every point of this interval.

Remark 1. Properties of the generalized derivative have been considered in[9].

Definition 3[9]. The set-valued mapping $X:\left[t_{0}, T\right] \rightarrow$ $\operatorname{conv}\left(R^{n}\right)$ is called absolutely continuous on the interval $\left[t_{0}, T\right]$ if there exist a measurable set-valued mapping $G(t)$ and a system of intervals $\left[t_{i}, t_{i+1}\right], i=0, . ., m, t_{m+1}=T$ such that for all $t \in\left[t_{i}, t_{i+1}\right], \quad i=0, . ., m$

$$
X(t)=X\left(t_{i}\right) \frac{h}{t} \int_{t_{i}}^{t} G(s) d s \text { or } X(t)=X\left(t_{i}\right)+\int_{t_{i}}^{t} G(s) d s .
$$

Theorem 1[9]. Let a set-valued mapping $X:\left[t_{0}, T\right] \rightarrow$ $\operatorname{conv}\left(R^{n}\right)$ is absolutely continuous on the interval $\left[t_{0}, T\right]$.

Then the set-valued mapping $X(\cdot)$ is differentiable in the generalized sense almost everywhere on the interval $\left[t_{0}, T\right]$ and $D X(t)=G(t)$ almost everywhere on $\left[t_{0}, T\right]$.

\section{Generalized Differential Equations with the Generalized Derivative}

First consider a differential equation with the generalized derivative that is similar to a differential equation with the Hukuhara derivative, i.e.

$$
D X=F(t, X), \quad X\left(t_{0}\right)=X_{0},
$$

where $D X(t)$ is the generalized derivative of a set-valued mapping $X:\left[t_{0}, T\right] \rightarrow \operatorname{conv}\left(R^{n}\right), \quad F:\left[t_{0}, T\right] \times \operatorname{conv}\left(R^{n}\right) \rightarrow \operatorname{conv}\left(R^{n}\right)$ is a set-valued mapping, $X_{0} \in \operatorname{conv}\left(R^{n}\right)$.

Definition 4. A set-valued mapping $X:\left[t_{0}, T\right] \rightarrow \operatorname{conv}\left(R^{n}\right)$ is said to be solution of differential equation (9) if it is absolutely continuous and satisfies (9) almost everywhere on $\left[t_{0}, T\right]$.

Remark 2. Unlike the case of differential equations with Hukuhara derivative, if a differential equation with the generalized derivative (9) has a solution then there exists an infinite number of solutions irrespective of the conditions on the right-hand side of the equation.

Example 1. Consider the following differential equation with the generalized derivative

$$
D X=[-1,1], \quad X(0)=[-2,2] \text {. }
$$

It is easy to check that the following set-valued mappings are the solutions of equation (10):

$$
\begin{gathered}
X_{1}(t)=[-2-t, 2+t], t \in[0,1], \\
X_{2}(t)=[-2+t, 2-t], t \in[0,1], \\
X_{3}(t)=\left\{\begin{array}{cc}
{[-2-t, 2+t],} & t \in[0,0.5], \\
{[-2.5+t, 2.5-t],} & t \in[0.5,1],
\end{array}\right. \\
X_{4}(t)=\left\{\begin{array}{cc}
{[-2,+t, 2-t],} & t \in[0,0.25], \\
{[-1.5-t, 1.5+t],} & t \in[0.25,0.5], \\
{[-2.5+t, 2.5-t],} & t \in[0.5,1] .
\end{array}\right.
\end{gathered}
$$

Also it is possible to construct other solutions, thus only $X_{1}(\cdot)$ will be the solution of the corresponding differential equation with the Hukuhara derivative

$$
D_{H} X=[-1,1], \quad X(0)=[-2,2]
$$

and $X_{1}(\cdot)$ and $X_{2}(\cdot)$ are solutions of the differential equation with the generalized derivative (in the sense of [8]).

Therefore we will consider the other differential equation with the generalized derivative:

$$
\begin{aligned}
& D X(t) \stackrel{h}{\Phi}(-\phi(t)) F_{1}(t, X(t))=\Phi(\phi(t)) F_{2}(t, X(t)), \\
& X\left(t_{0}\right)=X_{0},
\end{aligned}
$$

where $t \in\left[t_{0}, T\right] \quad ; \quad X:\left[t_{0}, T\right] \rightarrow \operatorname{conv}\left(R^{n}\right) \quad ; \quad X_{0} \in \operatorname{conv}\left(R^{n}\right) \quad ;$ $F_{1}, F_{2}:\left[t_{0}, T\right] \times \operatorname{conv}\left(R^{n}\right) \rightarrow \operatorname{conv}\left(R^{n}\right)$ are set-valued mappings; $\phi:\left[t_{0}, T\right] \rightarrow R$ is a continuous function; function $\Phi(\phi)= \begin{cases}1, & \phi>0, \\ 0, & \phi \leq 0 .\end{cases}$

Definition 5. A set-valued mapping $X:\left[t_{0}, T\right] \rightarrow \operatorname{conv}\left(R^{n}\right)$ is called the solution of differential equation (11) if it is continuous and on any subinterval $\left[\tau_{1}, \tau_{2}\right] \subset\left[t_{0}, T\right]$, where function $\phi(t)$ of constant signs, satisfies the integral equation

$$
X(t)+\int_{\tau_{1}}^{t} \Phi(-\phi(s)) F_{1}(s, X(s)) d s=X\left(\tau_{1}\right)+\int_{\tau_{1}}^{t} \Phi(\phi(s)) F_{2}(s, X(s)) d s .
$$

If on the interval $\left[\tau_{1}, \tau_{2}\right]$ the function $\phi(t)>0$, then $X(t)$ 
satisfies the integral equation

$$
X(t)=X\left(\tau_{1}\right)+\int_{\tau_{1}}^{t} F_{2}(s, X(s)) d s
$$

for $t \in\left[\tau_{1}, \tau_{2}\right]$ and $\operatorname{diam} X(t)$ increases.

If on the interval $\left[\tau_{1}, \tau_{2}\right]$ the function $\phi(t)<0$, then we have

$$
X(t)+\int_{\tau_{1}}^{t} F_{1}(s, X(s)) d s=X\left(\tau_{1}\right),
$$

i.e. $X(t)=X\left(\tau_{1}\right) \stackrel{h}{\int_{\tau_{1}}^{t}} F_{1}(s, X(s)) d s$ and $\operatorname{diam} X(t)$ decreases.

If on the interval $\left[\tau_{1}, \tau_{2}\right]$ the function $\phi(t) \equiv 0$, then we have $X(t) \equiv X\left(\tau_{1}\right)$.

So we can enter the other equivalent definition of a solution of equation (11).

Definition 6. A set-valued mapping $X:\left[t_{0}, T\right] \rightarrow \operatorname{conv}\left(R^{n}\right)$ is called the solution of differential equation (11) if it is absolutely continuous, satisfies (11) almost everywhere on $\left[t_{0}, T\right]$ and

$$
\operatorname{diam} X(t)=\left\{\begin{array}{c}
\text { increases if } \phi(t)>0, \\
\text { is constant if } \phi(t)=0, \\
\text { decreases if } \phi(t)<0 .
\end{array}\right.
$$

Example 2. Consider the following differential equation with generalized derivative

$$
D X \stackrel{h}{-} \Phi(-\sin t)[-2,4]=\Phi(\sin t)[1,3], X(0)=[2,4] .
$$

As $\sin t>0$ for $t \in(0, \pi)$ we have

$$
X(t)=[2,4]+\int_{0}^{t}[1,3] d s=[2,4]+[t, 3 t]=[2+t, 4+3 t]
$$

for $t \in[0, \pi]$.

So for $t=\pi$ we get $X(\pi)=[2+\pi, 4+3 \pi]$.

Further as $\sin t<0$ for $t \in(\pi, 2 \pi)$ we have

$$
\begin{gathered}
X(t)=[2+\pi, 4+3 \pi]-\int_{\pi}^{h}[-2,4] d s= \\
=[2+\pi, 4+3 \pi]-\int_{\pi}^{t} d s[-2,4]= \\
=[2+\pi, 4+3 \pi]-(t-\pi)[-2,4]= \\
=[2-\pi+2 t, 4+7 \pi-4 t] .
\end{gathered}
$$

So for $t=\frac{1+4 \pi}{3}<2 \pi$ we get $X\left(\frac{1+4 \pi}{3}\right)=\left\{\frac{8+5 \pi}{3}\right\}$.

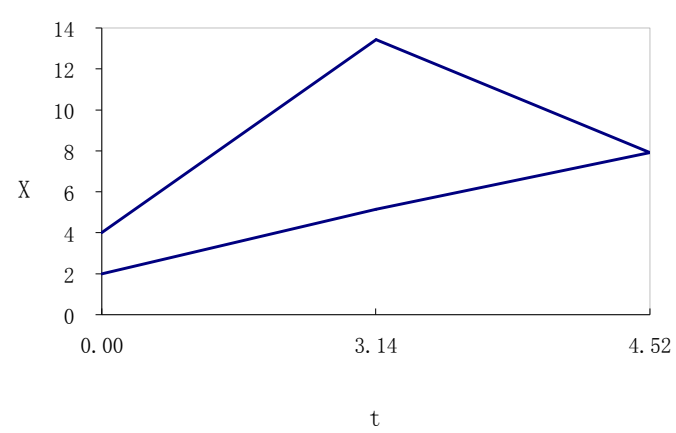

Figure 1. The graph of a solution of system (12)
It means that the solution exists only for $t \in\left[0, \frac{1+4 \pi}{3}\right]$ (see fig. 1).

Example 3. Consider the same differential equation with generalized derivative but with $\varphi(t)=-\sin (10 t)$ :

$$
D X \stackrel{h}{-} \Phi(\sin 10 t)[-2,4]=\Phi(-\sin 10 t)[1,3], X(0)=[2,4]
$$

As $-\sin 10 t<0$ for $t \in\left(0, \frac{\pi}{10}\right)$ then we have

$$
X(t)=[2,4]-\int_{0}^{t}[-2,4]=[2+2 t, 4-4 t] \text { for } t \in\left[0, \frac{\pi}{10}\right] .
$$

Further as $-\sin 10 t>0$ for $t \in\left(\frac{\pi}{10}, \frac{\pi}{5}\right)$ then we get

$$
X(t)=\left[2+\frac{\pi}{10}, 4-\frac{2 \pi}{5}\right]+\int_{\frac{\pi}{10}}^{t}[1,3] d s=\left[2+t, 4-\frac{7 \pi}{10}+3 t\right] .
$$

Further as $-\sin 10 t<0$ for $t \in\left(\frac{\pi}{5}, \frac{3 \pi}{10}\right)$ then we get

$$
X(t)=\left[2+\frac{\pi}{5}, 4-\frac{\pi}{10}\right]-\int_{\frac{\pi}{5}}^{t}[-2,4] d s=\left[2-\frac{\pi}{5}+2 t, 4+\frac{7 \pi}{10}-4 t\right] .
$$

So for $t=\frac{1}{3}+\frac{3 \pi}{20}<\frac{3 \pi}{10}$ we have $X\left(\frac{1}{3}+\frac{3 \pi}{20}\right)=\left\{\frac{8}{3}-\frac{\pi}{10}\right\}$.

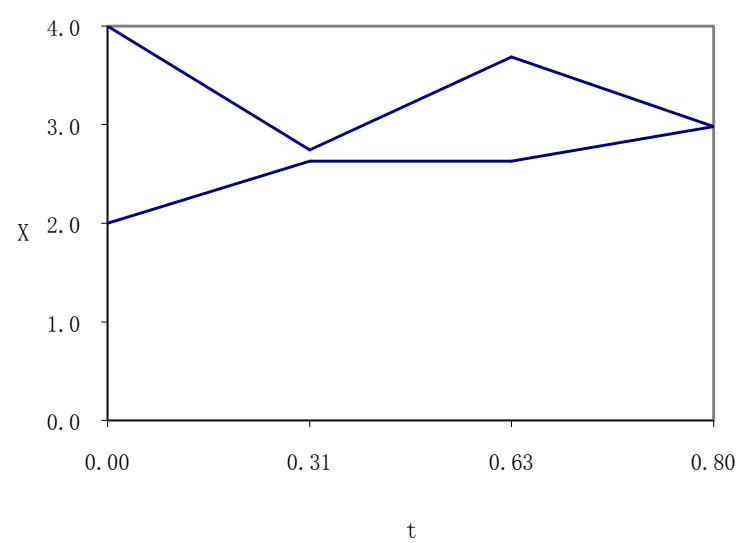

Figure 2. The graph of a solution of system (13)

It means that the solution exists only for $t \in\left[0, \frac{1}{3}+\frac{3 \pi}{20}\right]$ (see fig. 2).

Remark 3. It is obvious that the mappings $F_{1}(t, X)$, $F_{2}(t, X)$ define only on "how much" the mapping $X(\cdot)$ changes in case of its "decrease" $\left(F_{1}(t, X)\right)$ or "increase" $\left(F_{2}(t, X)\right)$ and function $\phi(\cdot)$ defines what will occur to $X(\cdot)$ ["decrease" or "increase"]. If $\phi(t) \equiv 0$ irrespective of $F_{1}(t, X(t))$ and $F_{2}(t, X(t))$ the mapping $X(\cdot)$ will be constant.

Example 4. Consider the differential equation from Example 2 with $\phi(t) \equiv 0$ for $t \in[0,2 \pi]$. Then $X(t) \equiv[2,4]$ for $t \in[0,2 \pi]$.

Remark 4. If we take $\phi(t) \equiv-1$ then we will have

$$
X(t)=[2,4]-\int_{0}^{h}[-2,4] d s=[2+2 t, 4-4 t] .
$$

Then for $t=\frac{1}{3}$ we get $X\left(\frac{1}{3}\right)=\left\{\frac{8}{3}\right\}$. So the solution exists 
for $t \in\left[0, \frac{1}{3}\right]$ (see fig. 3 ).

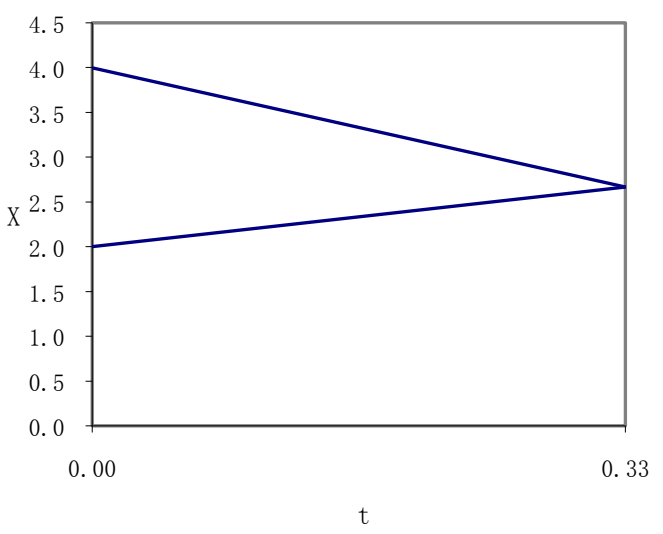

Figure 3. The graph of a solution of system (12) for $\varphi(t) \equiv-1$

So for all $\phi(t)$ we can guarantee the existence of solution of the differential equation on the interval $\left[0, \frac{1}{3}\right]$.

$$
D X(t) \stackrel{h}{-} \Phi(-\phi(t))[-2,4]=\Phi(\phi(t))[1,3], X(0)=[2,4]
$$

Let $C C\left(R^{n}\right) \quad(n \geq 2)$ be a space of all nonempty strictly convex closed sets of $R^{n}$ and all element of $R^{n}$ [27].

The following theorem of existence of the solution of equation (11) for case $C C\left(R^{n}\right)$ holds:

Theorem 2. Let the set-valued mappings $F_{1}(t, X)$, $F_{2}(t, X): R \times C C\left(R^{n}\right) \rightarrow C C\left(R^{n}\right)$ in the domain

$Q=\left\{(t, X) \in R \times C C\left(R^{n}\right): t \in\left[t_{0}, t_{0}+a\right], h\left(X, X_{0}\right) \leq b\right\}$ satisfy the following conditions:

i) for any fixed $X$ the set-valued mappings $F_{1}(\cdot, X)$, $F_{2}(\cdot, X)$ are measurable;

ii) for almost every fixed $t$ the set-valued mappings $F_{1}(t, \cdot)$, $F_{2}(t, \cdot)$ are continuous;

iii) $\left|F_{1}(t, X)\right| \leq m_{1}(t),\left|F_{2}(t, X)\right| \leq m_{2}(t)$, where $m_{1}(\cdot), m_{2}(\cdot)$ are summable on $t \in\left[t_{0}, t_{0}+a\right]$;

iv) $\phi(t)$ is continuous and has the finite number of intervals where $\operatorname{sign}(\phi(t))= \pm 1$,

v) int $X_{0} \neq \varnothing$.

Then there exists a solution of equation (11) defined on the interval $t \in\left[t_{0}, t_{0}+d\right]$, where $d>0$ satisfies the conditions

a) $d \leq a$;

b) $\varphi_{i}\left(t_{0}+d\right) \leq b$, where $\varphi_{i}(t)=\int_{0}^{t} m_{i}(s) d s, i=1,2$;

c) $\int_{\mu\left[t_{0}, t_{0}+d\right]} m_{1}(s) d s \leq \frac{\theta}{2}$

where $\theta=\min _{\|\psi\|=1}\left|C\left(X_{0}, \psi\right)-C\left(X_{0},-\psi\right)\right|$,

$C(X, \psi)=\max _{x \in X}\left(x_{1} \psi_{1}+\ldots+x_{n} \psi_{n}\right)$,

$\mu\left[t_{0}, t_{0}+d\right] \subset\left[t_{0}, t_{0}+d\right]: \phi(t)<0$ for $t \in \mu\left[t_{0}, t_{0}+d\right]$.

Proof. Let us consider some cases.

1) $\phi(t)>0$ for $t \in\left[t_{0}, t_{0}+a\right]$. Then equation (11) is the ordinary differential equation with Hukuhara derivative

$$
D_{H} X(t)=F_{2}(t, X), X\left(t_{0}\right)=X_{0} .
$$

Therefore, using[17] we get that the equation (11) has a solution $X(t)$ defined on $\left[t_{0}, t_{0}+d\right]$, where $d$ satisfies the condition $d=\min \{a, \gamma\}, \int_{t_{0}}^{t_{0}+\gamma} m_{2}(s) d s=b$.

2) $\phi(t) \equiv 0$ for $t \in\left[t_{0}, t_{0}+a\right]$. Then equation (11) is the ordinary differential equation with Hukuhara derivative $D X(t)=\{0\}, X\left(t_{0}\right)=X_{0}$ and therefore, $X(t) \equiv X_{0}$ is the solution of (11) on $\left[t_{0}, t_{0}+a\right]$.

3) $\phi(t)<0$ for $t \in\left[t_{0}, t_{0}+a\right]$. Then equation (11) is the equation with the generalized derivative

$$
D X(t) \stackrel{h}{-} F_{1}(t, X(t))=\{0\}, X\left(t_{0}\right)=X_{0} .
$$

According to Definition 5 consider the following integral equation

$$
X(t)=X_{0} \frac{h}{\int_{t_{0}}^{t}} F_{1}(s, X(s)) d s
$$

for $t \in\left[t_{0}, t_{0}+a\right]$ and prove the existence of solution on the some interval $\left[t_{0}, t_{0}+d\right]$.

3a) As $\left|F_{1}(t, X)\right| \leq m_{1}(t)$ for $(t, X) \in Q$, then

$$
F_{1}(t, X) \subset S_{m_{1}(t)}(0),
$$

where $S_{r}(a)=\left\{x \in R^{n}:\|x-a\| \leq r\right\}$.

$$
\begin{aligned}
& \text { So } \int_{t_{0}}^{t} F_{1}(s, X) d s \subset \int_{t_{0}}^{t} S_{m_{1}(s)}(0) d s=S_{\int_{t_{1}} m_{1}(s) d s}(0) . \\
& \text { Define by } S(t)=S_{\int_{t_{0}} m_{1}(s) d s}(0) .
\end{aligned}
$$

It is obviously, that if $t_{0}<t_{1}<t_{2}<t_{0}+a$, then

$$
\{0\}=S\left(t_{0}\right) \subset S\left(t_{1}\right) \subset S\left(t_{2}\right) \subset S\left(t_{0}+a\right) .
$$

As $X_{0} \in C C\left(R^{n}\right)$ and $\operatorname{int} X_{0} \neq \varnothing$, then there exists $d_{1}>0$ such that the set $S(t)$ can be embedded in the set $X_{0}$ for all $t \in\left[t_{0}, t_{0}+d_{1}\right]$ (i.e. there exists $\zeta(t)$ such that $S(t)+\zeta(t) \subset X_{0}$ ) and is not embedded for $t>t_{0}+d_{1}$. And, it is obviously, that $d_{1}$ can be found out from the equation $\int_{t_{0}}^{t_{0}+d_{1}} m_{1}(s) d s=\frac{\theta}{2}$.

Therefore, for all

$$
\begin{gathered}
(t, X) \in Q_{1}=\left\{(t, X) \in R \times C C\left(R^{n}\right):\right. \\
\left.t \in\left[t_{0}, t_{0}+d_{1}\right], h\left(X, X_{0}\right) \leq b\right\}
\end{gathered}
$$

the set $\int_{t_{0}}^{t} F_{1}(s, X) d s$ is embedded in the set $X_{0}$.

3b) As $F_{1}(t, X) \in C C\left(R^{n}\right)$ for all $(t, X) \in Q_{1}$, then $\int_{t_{0}}^{t} F_{1}(t, X) d s \in C C\left(R^{n}\right)$ for all $(t, X) \in Q$ [27]. Therefore, as $X_{0} \in C C\left(R^{n}\right)$ and that the set $S(t)$ can be embedded in the set $X_{0}$ for all $t \in\left[t_{0}, t_{0}+d_{1}\right]$, then the Hukuhara difference $X_{0}-\int_{t_{0}}^{t} F_{1}(s, X) d s$ exists for all $(t, X) \in Q_{1}[27]$.

$3 \mathrm{c})$ Let us find $d_{2}>0$ such that $\int_{t_{0}}^{t_{0}+d_{2}} m_{1}(s) d s=b$ and consider $d=\min \left\{a, d_{1}, d_{2}\right\}$.

3d) Choose any natural $k$. Sequentially on the intervals 


$$
t_{0}+i \Delta \leq t \leq t_{0}+(i+1) \Delta, \Delta=\frac{d}{k}, i=0, \ldots, k-1
$$

let us build the successive approximations of the solution

$$
\begin{gathered}
X^{k}(t)=X_{0} \text { for } t_{0}-\Delta \leq t \leq t_{0}, \\
X^{k}(t)=X_{0} \frac{h}{\int_{t_{0}}^{t}} F_{1}\left(s, X^{k}(s-\Delta)\right) d s \text { for } t \in\left[t_{0}, t_{0}+d\right]
\end{gathered}
$$

By 3b) $X^{k}(t)$ is exist and $X^{k}(t) \in C C\left(R^{n}\right)$ for all $k \in N$ and $t \in\left[t_{0}, t_{0}+d\right]$. Also by conditions i) and ii) of the theorem $X^{k}(t)$ is continuous on $\left[t_{0}, t_{0}+d\right]$ for all $k \in N$.

Besides

$$
\begin{gathered}
h\left(X^{k}(t), X_{0}\right)=h\left(X_{0}-\frac{h}{t_{0}} F_{1}\left(s, X^{k}(s-\Delta)\right) d s, X_{0}\right) \leq \\
\leq h\left(\{0\}, \int_{t_{0}}^{t} F_{1}\left(s, X^{k}(s-\Delta)\right) d s\right) \leq \int_{t_{0}}^{t} h\left(F_{1}\left(s, X^{k}(s-\Delta),\{0\}\right) d s \leq\right. \\
\leq \int_{t_{0}}^{t} m_{1}(s) d s \leq \varphi_{1}\left(t_{0}+d\right) \leq b .
\end{gathered}
$$

Hence, it follows that the sequence of the set-valued mappings $\left\{X^{k}(t)\right\}_{k=1}^{\infty}$ in uniformly bounded:

$$
h\left(X^{k}(t),\{0\}\right) \leq h\left(X_{0},\{0\}\right)+b .
$$

Let us show that the set-valued mappings $X^{k}(t)$ are equicontinuous. For any $\alpha<\beta \quad \alpha, \beta \in\left[t_{0}, t_{0}+d\right]$ and any natural $k$ the inequality holds

$$
\begin{gathered}
h\left(X^{k}(\alpha), X^{k}(\beta)\right)= \\
=h\left(X_{0}-\int_{t_{0}}^{\alpha} F_{1}\left(s, X^{k}(s-\Delta)\right) d s, X_{0} \frac{h^{\beta}}{t_{0}} F_{1}\left(s, X^{k}(s-\Delta)\right) d s\right)= \\
=h\left(\int_{\alpha}^{\beta} F_{1}\left(s, X^{k}(s-\Delta)\right) d s,\{0\}\right) \leq \int_{\alpha}^{\beta} h\left(F_{1}\left(s, X^{k}(s-\Delta)\right),\{0\}\right) d s \leq \\
\leq \int_{\alpha}^{\beta} m_{1}(s) d s=\varphi_{1}(\beta)-\varphi_{1}(\alpha)
\end{gathered}
$$

The function $\varphi_{1}(t)$ is absolutely continuous on $\left[t_{0}, t_{0}+d\right]$ as the integral of the summable function with a variable top limit. Hence, for any $\varepsilon>0$ there exists $\delta(\varepsilon)>0$ such that for all $\alpha, \beta$ such that $0 \leq \beta-\alpha<\delta$ the inequality $h\left(X^{k}(\alpha), X^{k}(\beta)\right)<\varepsilon$ is fair, the sequence $\left\{X^{k}(t)\right\}_{k=1}^{\infty}$ is equicontinuous.

According to Askoli theorem[28] we can choose a uniformly converging subsequence of the sequence $\left\{X^{k}(t)\right\}_{k=1}^{\infty}$. Its limit is a continuous set-valued mapping that we will denote by $X(t)$. As and the first summand is less than $\varepsilon$ for $\Delta=\frac{d}{k}<\delta$ in view of the equicontinuity of the set-valued mappings $\left\{X^{k}(t)\right\}_{k=1}^{\infty}$, then along the chosen subsequence $\left\{X^{k}(s-\Delta)\right\}_{k=1}^{\infty}$ converges to $X(t)$. Owing to the theorem conditions in (15) it is possible to pass to the limit under the sign of the integral. We receive that the set-valued mapping $X(t)$ satisfies equation (16) and $X\left(t_{0}\right)=X_{0}$, i.e. $X(t)$ is the solution of (15) on the interval $\left[t_{0}, t_{0}+d\right]$.

$$
\begin{gathered}
h\left(X^{k}(s-\Delta), X(s)\right) \leq \\
\leq h\left(X^{k}(s-\Delta), X^{k}(s)\right)+h\left(X^{k}(s), X(s)\right),
\end{gathered}
$$

4) In case when the function $\phi(t)$ changes sign on the interval $\left[t_{0}, t_{0}+a\right]$, the existence of the solution is proved combining cases 1)-3). The theorem is proved.

Theorem 3. Let the set-valued mappings $F_{1}(t, X)$, $F_{2}(t, X): R \times C C\left(R^{n}\right) \rightarrow C C\left(R^{n}\right)$ in the domain

$$
Q=\left\{(t, X) \in R \times C C\left(R^{n}\right): t \in\left[t_{0}, t_{0}+a\right], h\left(X, X_{0}\right) \leq b\right\}
$$

satisfy the conditions of Theorem 2 and satisfy the conditions

$$
\begin{aligned}
& h\left(F_{1}\left(t, X^{\prime}\right), F_{1}\left(t, X^{\prime \prime}\right)\right) \leq L_{1} h\left(X^{\prime}, X^{\prime \prime}\right), \\
& h\left(F_{2}\left(t, X^{\prime}\right), F_{2}\left(t, X^{\prime \prime}\right)\right) \leq L_{2} h\left(X^{\prime}, X^{\prime \prime}\right)
\end{aligned}
$$

for all $\left(t, X^{\prime}\right),\left(t, X^{\prime \prime}\right) \in Q$.

Then there exists the unique solution of equation (11) defined on the interval $t \in\left[t_{0}, t_{0}+d\right]$.

The proof is similar to[17,24].

Finally we consider example for case $C C\left(R^{2}\right)$.

Example 6. Consider the following differential equation with generalized derivative

$$
D X \stackrel{h}{-} \Phi(t-1) X=\Phi(1-t) \frac{1}{2} X, X(0)=S_{1}(0),
$$

where $X: R \rightarrow C C\left(R^{2}\right)$.

It is obvious that $X(t)=\left\{\begin{array}{cc}S_{e^{\frac{1}{2}}}(0), & t \leq 1, \\ S_{e^{1,-t}}(0), & t>1\end{array}\right.$ is the solution of differential equation (18) (see fig. 4).

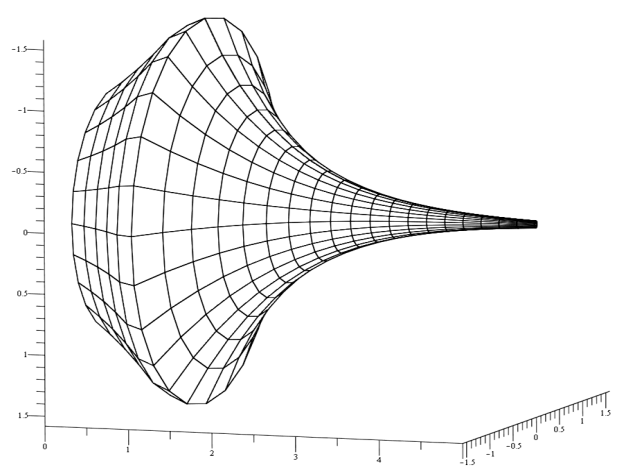

Figure 4. The graph of a solution of system (18)

Remark 5. Also it is possible to prove the similar results if $C C\left(R^{n}\right)$ be a space of all nonempty M- strongly convex closed sets of $R^{n}$ and all element of $R^{n}$ [29].

Remark 6. Let's notice that we considered some continuous function $\phi(t)$ but it is also possible to take $\phi(t, X)=\operatorname{diam} X(t)-c(t)$, for example, where $c(t)$ - is the diameter of some etalon set-valued mapping.

\section{Conclusions}

In this paper the concept of generalized differentiability (proposed in[9]) for set-valued mappings is used. The new type of the set-valued differential equation - generalized set differential equations - is considered. The existence and uniqueness theorems for set-valued differential equations 
with generalized derivative are proved.

\section{REFERENCES}

[1] Hukuhara, M., 1967, Integration des applications mesurables dont la valeur est un compact convexe, Funkcial. Ekvac., 10, 205-223.

[2] Bridgland, T.F., 1970, Trajectory integrals of set valued functions, Pacific J. of math., 33 (1), 43-68.

[3] Tyurin, Yu.N., 1965, Mathematical statement of the simplified model of industrial planning, Economic and mathematical methods, 1 (3), 391 - 409. (in Russian)

[4] Banks, H.T., and Jacobs, M.Q., 1970, A differential calculus for multifunctions, J. Math. Anal. Appl., 29, 246-272, doi:10.1016/0022-247X(70)90078-8.

[5] Plotnikov, A.V., 2000, Differentiation of multivalued mappings. T-derivative, Ukr. Math. J., 52 (8), 1282-1291, doi:10.1023/A:1010361206391.

[6] Plotnikov, V.A., Plotnikov, A.V., and Vityuk, A.N., 1999, Differential equations with a multivalued right-hand side. Asymptotic methods, AstroPrint, Odessa. (in Russian)

[7] Vityuk, A.N., 2003, Fractional differentiation of multivalued mappings, Dopov. Nats. Akad. Nauk Ukr. Math. Prirodozn. Tekh. Nauki, (10), 75-79. (in Russian)

[8] Bede, B., and Gal, S.G., 2005, Generalizations of the differentiability of fuzzy-number-valued functions with applications to fuzzy differential equations, Fuzzy Sets Syst., 151, 581-599. doi:10.1016/j.fss.2004.08.001.

[9] Plotnikov, A.V., and Skripnik, N.V., 2011, Set-valued differential equations with generalized derivative, J. Adv. Res. Pure Math., 3 (1), 144 - 160, doi:10.5373/jarpm.475.062210.

[10] Bede, B., and Stefanini, L., 2009, Numerical Solution of Interval Differential Equations with Generalized Hukuhara Differentiability, IFSA/EUSFLAT Conf., 730-735.

[11] Bede, B., and Stefanini L., 2008, Generalized Hukuhara differentiability of interval-valued functions and interval differential equations, Working Paper Series in Economics, Math. and Statistics, WP-EMS \#2008/03, Univ. Urbino "Carlo Bo".

[12] de Blasi, F.S., and Iervolino F., 1969, Equazioni differentiali con soluzioni a valore compatto convesso, Boll. Unione Mat. Ital., 2 (4-5), 491-501.

[13] Chalco-Cano, Y., Román-Flores, H., and Jiménez-Gamero, M.D., 2011, Generalized derivative and $\pi$-derivative for set-valued functions, Inf. Sci., 181 (1), 2177-2188, doi:10.1016/j.ins.2011.01.023.

[14] Lasota, A., and Strauss, A., 1971, Asymptotic behavior for differential equations which cannot be locally linearized,
Journal of Differential Equations, (10), 152-172, doi:10.1016/0022-0396(71)90103-3.

[15] Martelli, M., and Vignoli, A., 1974, On differentiability of multi-valued maps, Boll. Unione Mat. Ital., 4 (10), 701-712.

[16] Plotnikova, N.V., 2005, Systems of linear differential equations with a $\pi$-derivative and linear differential inclusions, Russ. Acad. Sci., Sb., Math., 196 (11-12), 1677-1691, doi:10.1070/SM2005v196n11ABEH003726.

[17] Plotnikov, A.V., and Skripnik, N.V., 2009, Differential equations with "clear" and fuzzy multivalued right-hand sides. Asymptotics Methods, AstroPrint, Odessa. (in Russian)

[18] Stefanini, L., and Bede, B., 2009, Generalized Hukuhara differentiability of interval-valued functions and interval differential equations, Nonlinear Anal., Theory Methods Appl., Ser. A, Theory Methods, 71 (3-4), 1311-1328, doi: 10.1016/j.na.2008.12.005.

[19] de Blasi, F.S., and Iervolino F., 1971, Euler method for differential equations with set - valued solutions, Boll. Unione Mat. Ital., 4 (4), 941-949.

[20] de Blasi, F.S., Lakshmikantham, V., and Gnana Bhaskar T., 2007, An existence theorem for set differential inclusions in a semilinear metric space, Control Cybernet., 36 (3), 571-582.

[21] Brandao Lopes Pinto, A.J., de Blasi, F.S., and Iervolino, F., 1970, Uniqueness and existence theorems for differential equations with compact convex valued solutions, Boll. Unione Mat. Ital., 4, 534-538.

[22] Lakshmikantham, V., Granna Bhaskar, T., and Vasundhara Devi, J., 2006, Theory of set differential equations in metric spaces, Cambridge Scientific Publishers.

[23] Lakshmikantham, V., and Mohapatra, R.N., 2003, Theory of Fuzzy Differential Equations and Inclusions, Taylor \& Francis, London, doi:10.1201/9780203011386.

[24] Perestyuk, N. A., Plotnikov, V.A., Samoilenko, A.M., and Skripnik, N.V., 2011. Differential equations with impulse effects: multivalued right-hand sides with discontinuities, de Gruyter Stud. Math.: 40, Berlin/Boston: Walter De Gruyter GmbH\&Co.

[25] Rådström, H., 1952, An embedding theorem for spaces of convex sets, Proc. Amer. Math. Soc., 3, 165-169.

[26] Filippov, A.F., 1988, Differential equations with discontinuous righthand sides, Kluwer Academic Publishers Group, Dordrecht.

[27] Polovinkin, E.S., 1996, Strongly convex analysis, Sb. Math., 187, 259-286, doi:10.1070/SM1996v187n02ABEH000111.

[28] Kelley, J.L., 1975, General topology. Reprint of the 1955 edition[Van Nostrand, Toronto, Ont.]. Graduate Texts in Mathematics, No. 27. Springer-Verlag, New York-Berlin.

[29] Balashov, M.V., Polovinkin, E.S., 2000, M-strongly convex subsets and their generating sets, Sb. Math., 191, 25-60, doi:10.1070/sm2000v191n01ABEH000447. 